\title{
Enhanced fetoplacental angiogenesis in pre-gestational diabetes mellitus: the extra growth is exclusively longitudinal and not accompanied by microvascular remodelling
}

\author{
T. M. Mayhew \\ School of Biomedical Sciences, E Floor, Queen's Medical Centre, University of Nottingham, United Kingdom
}

\begin{abstract}
Aims/hypothesis. Morphometric studies on pregnancies complicated by gestational diabetes mellitus have found no evidence of increased fetoplacental angiogenesis. Here, placentas from control subjects and patients with pre-gestational Type I (insulin-dependent) diabetes mellitus were used to test for differences in measures of angiogenesis and vascular remodelling.

Methods. Term placentas were collected from non-diabetic subjects and well-controlled diabetic patients grouped according to duration and severity into White classes B, C, D and F/R. Tissues were obtained by uniform random sampling for position and orientation. Volumes, surface areas and lengths of peripheral villi and their capillaries were estimated stereologically. Comparisons were drawn by analysis of variance and used to interpret mechanisms of growth, villous capillarization and vessel remodelling.

Results. Placentas associated with all classes of diabetes contained greater (19-45\%) volumes of fetal
\end{abstract}

capillaries attributable solely to increases in the combined length of capillaries (12-47\%) and not to alteration of vessel cross-sectional area or perimeter. Longitudinal growth tended to increase capillarization (capillary:villus length ratios up to $19 \%$ larger) but changes were inconsistent between diabetic classes. There was no evidence of altered vascular remodelling (cross-sectional shape factors, perimeter ${ }^{2} /$ area, were preserved).

Conclusions/interpretations. In well-controlled pre-gestational diabetes, fetoplacental angiogenesis is enhanced and occurs exclusively by longitudinal growth. Differences may involve hypoxic or other metabolic effects on endothelial cells, perivascular cells and angiogenic factors. The findings differ from those previously reported in gestational diabetes. No differences were associated exclusively with the presence of diabetic complications. [Diabetologia (2002) 45:1434-1439]

Keywords Placenta, pre-gestational diabetes, capillaries, angiogenesis, remodelling.
Received: 17 April 2002 / Revised: 17 June 2002

Published online: 5 September 2002

C) Springer-Verlag 2002

Corresponding author: Dr. T. M. Mayhew, School of Biomedical Sciences, E Floor, Queen's Medical Centre, University of Nottingham, Nottingham NG7 2UH, UK. E-mail: terry.mayhew@nottingham.ac.uk

Abbreviations: A, Mean cross-sectional area of capillaries; Ang, angiopoietin; FGF, fibroblast growth factor; GDM, gestational diabetes mellitus; $\mathrm{P}$, mean cross-sectional perimeter of capillaries; PlGF, placenta growth factor; pre-GDM, pregestational diabetes mellitus; VEGF, vascular endothelial growth factor.
Diabetes mellitus is a major pregnancy complication associated with increased fetal morbidity and mortality linked to hypoxic stress, hyperglycaemia and various other metabolic abnormalities [1, 2, 3]. Less clear is the scale of change within the placenta, and yet an important factor influencing transport to the fetus is integrated development of villi and fetal vessels within them. Hypoxia-dependent foetoplacental angiogenesis during normal gestation helps to determine the pattern and scale of villous growth and maturation by regulating angiogenic growth factors and their natural receptors and antagonists $[4,5,6,7,8]$.

Early in gestation (before 20 weeks), hypoxia upregulates angiogenic factors (notably, vascular endo- 
thelial growth factor, VEGF, and angiopoietin-2, Ang-2) and down-regulates or destabilizes anti-angiogenic factors (e.g. angiopoietin-1, Ang-1). Expression of ligand receptors also alters. As a consequence, endothelial cells proliferate and tightly-looped fetal capillaries develop by branching angiogenesis $[4,5,6$, $7,8]$. Later, oxygen tensions rise and a switch downregulates VEGF, up-regulates placenta growth factor (PlGF) and shifts the Ang-2 to Ang-1 mRNA ratio in favour of Ang-1. The result is non-branching angiogenesis in which capillaries grow from existing vessels by increases in length. These events are accompanied by altered interactions between endothelial cells and perivascular cells, principally pericytes but also smooth muscle cells $[8,9,10]$. These interactions also affect endothelial proliferation and vessel maturation and stabilization [11, 12, 13].

In pregnancies complicated by fetal hypoxia, different categories of hypoxia can be identified based on observed patterns of angiogenesis and villous development [5] and companion changes in growth factors associated with villous macrophages, trophoblast and capillary endothelium $[5,14,15,16,17]$. In preplacental hypoxia, the mother, placenta and fetus are hypoxic, there is excessive branching angiogenesis and growth of capillaries and villi is impoverished. This scenario accompanies maternal anaemia and pregnancy at high altitude $[18,19,20]$. In uteroplacental hypoxia, blood delivery to the maternal intervillous space is reduced and leads to more heterogeneous development of villi with a shift towards branching angiogenesis. This picture is seen in pre-eclampsia and associated with a reduced total length, but conserved total volume, of capillaries $[6,20]$. In postplacental hypoxia, the mother is normoxic but the fetus is hypoxic and the placenta experiences 'relative hyperoxia' [21]. This favours non-branching angiogenesis and occurs in intrauterine growth restriction with abnormal end-diastolic flow in umbilical arteries.

Superficially, placental adaptations in pre-gestational diabetes mellitus (pre-GDM) resemble those in postplacental hypoxia. For example, maternal haematocrit is normal but there are increased fetal haematocrits, haemoglobin concentrations and concentrations of erythropoiesis [22, 23, 24]. In addition, changes in oxygen diffusive conductances affect principally the downstream tissues (villous stroma and fetal capillaries) of the intervascular barrier [23]. Earlier studies on groups of pre-GDM patients suggested that there is increased angiogenesis and increased capillarization of villi [24]. In contrast, recent studies have shown that angiogenesis is unperturbed in cases of gestational diabetes (GDM) [25].

Immunochemical studies on pre-GDM placentas have failed to detect differences in staining intensity or localization of VEGF or VEGF receptors-2 and -3 but noted inconsistent increases in VEGF receptor-1 intensity [16]. Concentrations of PlGF in maternal se- rum were normal. Interestingly, increased PIGF expressions and local VEGF concentrations accompany retinal neovascularization in diabetic patients with proliferative retinopathy $[26,27,28]$. Also, angiogenesis and VEGF expression are influenced by concentrations of blood glucose and advanced glycation endproducts (AGEs) [29, 30, 31, 32].

The aim of this study was to undertake a more detailed analysis of different classes of insulin-dependent pre-GDM placentas to test for quantitative histological evidence of angiogenesis and vascular remodelling. Archival material from well-controlled pregnancies varying in onset, duration, severity and degree of complication was studied.

\section{Materials and methods}

Provenance and classification of subjects. Material from 68 pregnancies was examined [23, 24]. Background details included anthropometric, clinical and haematological data together with maternal age, socio-economic status, reproductive history and volunteered smoking status. Non-hypertensive diabetic women were referred at 8-12 weeks of gestation and classified on White criteria [33, 34]: $n=10$ in class B, $n=7$ class $\mathrm{C}, n=11$ class $\mathrm{D}$ and $n=6$ class $\mathrm{F} / \mathrm{R}$. All diabetic patients were insulin-dependent but differed in onset (B after 20, C at 10-20, $\mathrm{D}$ at $0-10$ years of age respectively), duration $(\mathrm{B}<10, \mathrm{C} 10-20$ and $\mathrm{D}>20$ years respectively) and presence of complications ( $\mathrm{F}$ with diabetic nephropathy, $\mathrm{R}$ with proliferative retinopathy). Another group of 34 subjects served as control subjects who were healthy women completing uncomplicated pregnancies.

Blood analyses included glucose, haemoglobin and glycated haemoglobin $\left(\mathrm{HbA}_{1 \mathrm{c}}\right)$. After examinations, insulin dosages were recommended individually and patients monitored at 2-week intervals throughout the pregnancy. Diabetic patients were admitted to hospital 3 to 4 weeks before term when physical and other examinations were repeated. The aim was to maintain mean blood glucose concentrations at $6 \mathrm{mmol} / \mathrm{l}$ and $\mathrm{HbA}_{1 \mathrm{c}}$ at less than $7 \%$ of total haemoglobin. Achieved values were as follows: class $\mathrm{B}$ (glucose $5.9 \mathrm{mmol} / \mathrm{l} ; \mathrm{HbA}_{1 \mathrm{c}} 6.1 \%$ ), class $\mathrm{C}$ (glucose $5.9 \mathrm{mmol} / \mathrm{l} ; \mathrm{HbA}_{1 \mathrm{c}} 6.4 \%$ ), classes D and F/R (glucose $8.7 \mathrm{mmol} / \mathrm{l}$; $\mathrm{HbA}_{1 \mathrm{c}} 6.8 \%$ ). Newborn babies without congenital malformations were delivered at 37 to 42 weeks (judged by the last date of menstruation, clinical examination and ultrasound).

Tissue preparation and sampling. Umbilical cords were ligatured and cut within $30 \mathrm{~s}$ of delivery. Placentas were weighed and immersion-fixed in formalin. For stereological estimation of tissue volumes and surface areas, systematic random samples of tissue were selected and embedded in paraffin wax after randomising tissue orientation [23, 24]. Blocks chosen randomly were sectioned at $4 \mu \mathrm{m}$ and stained by the Masson trichrome technique. Systematic random samples of microscopical fields were recorded and viewed as colour slide transparencies projected at $\times 2000$ magnification.

Stereological estimations. Test point, intersection and transection counting methods were used to estimate volumes, surface areas and lengths [35]. Estimates were confined to capillaries within peripheral (intermediate and terminal) villi and excluded larger vessels found in the trunks and primary branches of villous trees. As a measure of capillarization, a capillary:villus 
Table 1. Capillary growth (total volume, surface area, length), capillary size (mean perimeter, area) and shape (perimeter ${ }^{2} /$ area) in cross section and villous capillarization (capillary:villus length ratio) in human placentas. Values are means (SEM) for groups of control subjects and different White classes of insulin-dependent pregnant diabetic subjects

\begin{tabular}{|c|c|c|c|c|c|c|c|}
\hline Group, type, class & $\begin{array}{l}\text { Total } \\
\text { volume, } \\
\mathrm{cm}^{3}\end{array}$ & $\begin{array}{l}\text { Total } \\
\text { surface, } \\
\mathrm{m}^{2}\end{array}$ & $\begin{array}{l}\text { Total } \\
\text { length, } \\
\mathrm{km}\end{array}$ & $\begin{array}{l}\text { Mean area } \\
\text { in cross } \\
\text { section, } \\
\mu^{2}\end{array}$ & $\begin{array}{l}\text { Mean } \\
\text { perimeter in } \\
\text { cross section, } \\
\mu \mathrm{m}\end{array}$ & $\begin{array}{l}\text { Shape factor } \\
\text { in cross } \\
\text { section, } \\
\mu^{2} / \mu \mathrm{m}^{2}\end{array}$ & $\begin{array}{l}\text { Capillary: } \\
\text { villus length } \\
\text { ratio, } \\
\mathrm{km} / \mathrm{km}\end{array}$ \\
\hline Control subjects & $46.4(2.39)$ & $6.93(0.36)$ & $219(11.8)$ & $218(8.36)$ & $31.8(0.58)$ & $4.8(0.14)$ & $3.7(0.12)$ \\
\hline Type I, B patients & $65.6(9.83)$ & $9.89(1.13)$ & $323(27.6)$ & $199(16.5)$ & $30.3(1.27)$ & $4.7(0.22)$ & $4.2(0.18)$ \\
\hline Type I, C patients & $55.1(4.87)$ & $7.70(0.43)$ & $256(15.2)$ & $218(18.1)$ & $30.2(0.69)$ & $4.3(0.30)$ & $3.6(0.32)$ \\
\hline$p$ level & $p<0.01$ & $p<0.01$ & $p<0.01$ & NS & NS & NS & $p<0.05$ \\
\hline
\end{tabular}

Table 2. Capillary growth, capillary size and shape in cross section and villous capillarization in human placentas. Values are means (SEM) for groups of control subjects pregnancies and insulin-dependent pregnant diabetic patients without (classes $\mathrm{B}+\mathrm{C}$ ) and with (classes $\mathrm{D}+\mathrm{F}+\mathrm{R}$ ) vascular complications

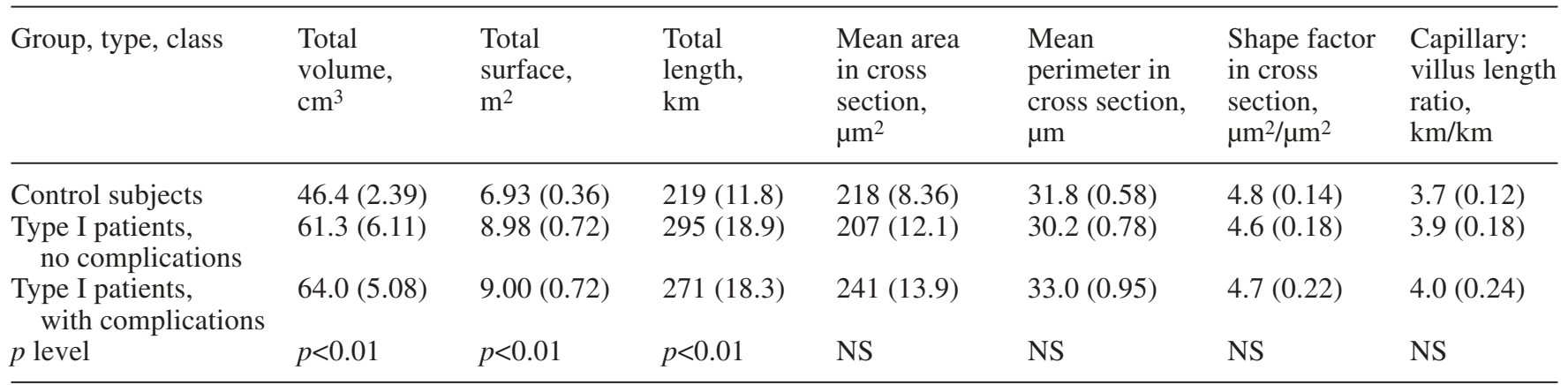

length ratio was calculated by dividing total capillary length by total length of peripheral villi. Mean cross-sectional areas $\left(\mathrm{A}\right.$, in $\left.\mu \mathrm{m}^{2}\right)$ of capillaries were calculated by dividing volumes by lengths and cross-sectional perimeters $(\mathrm{P}$, in $\mu \mathrm{m})$ by dividing surface areas by lengths. Subsequently, we calculated a dimensionless coefficient $\left(\mathrm{P}^{2} / \mathrm{A}, \mu \mathrm{m}^{2} / \mu \mathrm{m}^{2}\right)$ which reflects capillary shape in cross-section. This coefficient offers a convenient index of vascular remodelling.

Statistics. Group means and standard errors of means (SEM) were calculated and between-group differences examined by analysis of variance using statistical software (Unistat Ltd, London, UK). Null hypotheses were rejected with a probability level of less than 0.05 .

\section{Results}

In the control group, mean (SEM) gestational age was $39.5(0.2)$ weeks, birthweight $3.48(0.09) \mathrm{kg}$, placental volume 476 (16) $\mathrm{cm}^{3}$ and cord haemoglobin $9.3(0.23) \mathrm{mmol} / \mathrm{l}$. Diabetic subjects had higher levels of cord haemoglobin (variance ratio, $\mathrm{F}=3.75$; degrees of freedom, $\mathrm{df}=4,32 ; p<0.05)$ and shorter pregnancies by about 2 weeks but there were no differences in birthweight or placental weight. Cord haemoglobin concentrations did not vary between classes of diabetes. Measures of angiogenesis (capillary volume, surface area, length), vascular remodelling (crosssectional area, perimeter, shape factor) and villous capillarization (capillary:villus length ratio) are summarised in Table 1. Data were re-analysed after distinguishing between patients with and without vascular complications (Table 2). For this purpose, patients in White classes B and C were pooled (they showed no evident vascular pathology) as were classes F and R (evident complications) and D (intermediate). This secondary analysis did not alter findings substantively.

Fetoplacental angiogenesis. Non-diabetic placentas contained $46.4(2.39) \mathrm{cm}^{3}$ of capillaries with a surface area of $6.93(0.36) \mathrm{m}^{2}$ and a combined length of $219(11.8) \mathrm{km}$. All three variables were greater in diabetic placentas (volume: $\mathrm{F}=3.70 ; \mathrm{df}=4,63 ; p<0.01$; surface: $\mathrm{F}=4.20, p<0.01$; length: $\mathrm{F}=4.86 ; p<0.01)$ but there were no differences between diabetic groups.

Vascular remodelling. In control subjects, the mean cross-sectional area of capillaries was $218(8.36) \mu \mathrm{m}^{2}$, the perimeter $31.8(0.58) \mu \mathrm{m}$ and the shape factor $4.8(0.14) \mu \mathrm{m}^{2} / \mu \mathrm{m}^{2}$. No departures were detected in or between diabetic subjects.

Villous capillarization. The capillary:villus length ratio in control subjects was about $3.7(0.12) \mathrm{km} / \mathrm{km}$, equivalent to roughly two capillary loops per villus. In diabetic patients, the growth in length of capillaries tended to exceed the linear growth of villi and ratios 
increased by up to $19 \%(\mathrm{~F}=3.12 ; p<0.05)$. However, there were inconsistencies between diabetic classes and, when groups were distinguished by the presence (classes $\mathrm{D}+\mathrm{F}+\mathrm{R}$ ) or absence (classes $\mathrm{B}+\mathrm{C}$ ) of vascular complications, no differences in length ratios were detected.

\section{Discussion}

Increased fetoplacental angiogenesis occurs in pregnancies complicated by Type I pre-gestational diabetes despite good control of glycaemia and glycated haemoglobin $\mathrm{HbA}_{1 \mathrm{c}}$ values. The additional angiogenesis was not accompanied by changes in capillary calibre but was exclusively longitudinal. Nor was it accompanied by changes in capillary cross-sectional shape, suggesting there was no vascular remodelling beyond that in non-diabetic subjects. Finally, though not found consistently in all classes, villous capillarization tended to be greater in diabetic pregnancies. However, re-analysis of the data in diabetic women with or without complications failed to show differences between the groups. Due to the nature of changes during the last few weeks of normal gestation, the discrepancy between diabetic subjects and control subjects in terms of gestational age is likely to underestimate the magnitude of differences in capillary volume, surface and length and villous capillarization.

The changes in angiogenesis and capillarization are consistent with earlier findings on diabetic placentas [36, 37, 38]. Moreover, although another study [39] noted an increased incidence of hypovascularized villi in Type I diabetic patients, histometric counts of capillary and villous profiles [36, 37, 38] produced data consistent with the present differences in capillarization expressed by capillary:villus length ratios. Another deduction from these investigations $[36,37,38]$ is that the mean area of an endothelial squame (calculated by dividing capillary surface area by number of endothelial nuclei) is conserved in control subjects and diabetic subjects. This makes it likely that the longitudinal growth of capillaries occurring in diabetes is achieved by extra endothelial proliferation rather than cellular remodelling. This is consistent with the maintained cross-sectional calibre and shape of capillaries. It is emphasised that the term longitudinal growth refers to an increase in combined length of capillary vessels without accompanying changes in calibre and does not imply an altered pattern (branching or non-branching) of angiogenesis.

Although not providing direct evidence of fetal hypoxia, studies on diabetic pregnancies [22, 23, 24] have found high concentrations of cord haemoglobin, erythropoietin and erythropoiesis as well as improvements in oxygen diffusive conductances on the fetal side of the placenta. In our samples, cord haemoglobin concentrations were increased in diabetes (by about $10 \%$ ) but did not vary between diabetic classes. In other pregnancies complicated by preplacental, uteroplacental or postplacental hypoxia, changes occur in angiogenesis, vascular remodelling and capillarization. In maternal anaemia, villous hypercapillarization is not accompanied by increased angiogenesis or changes in vessel calibre [20, 40]. In fact, capillary volumes and lengths are reduced [20]. High-altitude pregnancy could lead to hypercapillarization and decreases in vessel calibre but variable responses in terms of capillary volume and length $[18,19,20]$. In pre-eclampsia, capillarization is not altered but capillary volume is essentially conserved because decreases in capillary length are compensated by increases in calibre [20]. In GDM, capillary volume, length and calibre do not vary [25]. Several conclusions could be drawn from these studies. Firstly, it is clearly dangerous to equate hypercapillarization with increased angiogenesis. Secondly, it is sensible to monitor absolute volume, length and calibre in order to interpret fully the nature of vascular change. Thirdly, capillary responses to pre-GDM do not mirror those seen in GDM or fall neatly into the patterns observed in other types of fetal hypoxia. A possible interpretation of the latter is that placental changes in pre-GDM are influenced by other confounding factors and do not represent responses solely to postplacental hypoxia.

Hypoxia up-regulates VEGF-A, a potent mitogen for endothelial cells and pericytes. Concentrations of VEGF-A are increased in patients with diabetic retinopathy and male patients with diabetic nephropathy [41]. Although caution is necessary when extrapolating angiogenic events from one tissue site to another, interrelations between hypoxia, growth factors and vascular cells have been extensively studied in diabetic retinopathy. In this context, the ability to form new retinal vessels depends on dissociation of pericytes from vascular endothelium and subjacent basal lamina $[10,11]$. Once this early phase of VEGF dependence ends, endothelial cells secrete a platelet-derived growth factor to trigger re-association with pericytes. This activates transforming growth factor- $\beta$ which stabilizes young vessels by inhibiting endothelial proliferation, promoting differentiation of perivascular cells and producing basal lamina and cell adhesion molecules $[10,12]$. Angiopoietins are also implicated in stabilizing young vessels. Ang-1 acts to stabilize vessels but low oxygen tensions up-regulate its antagonist, Ang-2 [10, 42]. In cultured human dermal microvascular endothelial cells, hypoxia also down-regulates endostatin, a potent anti-angiogenic factor found in endothelial cells and pericytes [8].

During normal gestation, branching angiogenesis in villi occurs in a hypoxic milieu and is regulated also by Ang-2 and VEGF [5, 6, 7]. As intervillous oxygen tensions rise, VEGF is down-regulated but PlGF and Ang-1 are up-regulated. Within villi, angiogenic fac- 
tors are associated with endothelium, trophoblast and stromal macrophages. As yet, detailed analyses on the relative incidences of pericytes around capillaries during gestation are lacking. Other growth factors could be implicated in the angiogenic changes during gestation. For example, fibroblast growth factor-2 (FGF-2 or basic FGF) is an endothelial cell mitogen expressed in placental villi, in maternal serum and peaking in the second trimester [43]. Values are greater in diabetic pregnancies and greatest in those complicated by retinopathy.

Amongst the metabolic complications of diabetes is hyperglycaemia and consequential non-enzymic glycation of proteins (including basal lamina proteins) and production of AGEs. Hyperglycaemia in vitro has similar anti-proliferative effects whether endothelial cells are obtained from microvessels or larger vessels [44, 45]. Excessive glycation of extracellular matrix produced by human umbilical vein endothelial cells, cultured in high concentrations of glucose, might explain impaired adhesion of pericytes subsequently grown on matrices [46]. AGEs increase VEGF expression in the rat retina and act synergistically with hypoxia [30]. The effects of glycated albumin on VEGF expression in the eyes are also additive with insulin when injected intraperitoneally in mice [31]. AGE receptors are in various cells including endothelial cells, pericytes, smooth muscle cells and macrophages. AGEs are toxic to pericytes and chorioallantoic membrane assays have shown that glycated albumin induces angiogenesis but the young vessels lack pericytes [32].

It is possible that increased fetoplacental angiogenesis confirmed here in pre-GDM arises following hyperglycaemic episodes at early stages of pregnancy (before mid-gestation) when oxygen tensions are low, VEGF is up-regulated and branching angiogenesis is dominant. At this period, transiently high glucose concentrations might act additively with low oxygen to increase VEGF expression even further. They might also have direct toxic effects on pericytes as well as influencing their association with endothelial cells via pericyte adhesion to basal lamina. These events would delay further the stabilization of young capillaries. After mid-gestation, the effects of high glucose might diminish since the period of non-branching angiogenesis coincides with elevated oxygen tensions and PIGF levels and down-regulation of VEGF.

An intriguing further question remains: why is fetoplacental angiogenesis not increased in GDM? Could it be that the metabolic disturbance identified above do not appear in GDM until after the critical period of branching angiogenesis has passed? GDM is usually diagnosed in the second half of pregnancy. To address these issues, investigations of control and diabetic placentas at different periods of gestation are required. In Type I diabetic pregnancies, placental morphology is correlated with the degree of metabolic control up to
32 weeks of gestation but not thereafter [39]. This emphasises the importance of recording the quality of glycaemic control at various stages of pregnancy. But the same token, monitoring VEGF levels only at term has failed to show differences in diabetic subjects [16] but this does not allow conclusions to be drawn about events at early stages of pregnancy and particularly before mid-gestation when VEGF-dependent angiogenesis is known to occur.

Acknowledgements. I thank Drs M.R. Jackson (Toronto), J. Klebe and F.B. Sørensen (Aarhus) for the placental material on which analyses were based and the Special Trustees of Nottingham University Hospitals for funds to purchase a microscope for random tissue sampling.

\section{References}

1. Kuhl C, Hornes PJ, Anderson O (1985) Etiology and pathophysiology of gestational diabetes mellitus. Diabetes 34 [Suppl 2]: 66-70

2. Desoye G, Shafrir E (1996) The human placenta in diabetic pregnancy. Diabetes Rev 4: 70-89

3. Casson IF, Clarke CA, Howard CV et al. (1997) Outcomes of pregnancy in insulin dependent diabetic women: results of a five year population cohort study. BMJ 315: 275-278

4. Clark DE, Smith SK, Sharkey AM, Charnock-Jones DS (1996) Localization of VEGF and expression of its receptors flt and KDR in human placenta throughout pregnancy. Hum Reprod 11: 1090-1098

5. Kingdom JCP, Kaufmann P (1997) Oxygen and placental villous development: origins of foetal hypoxia. Placenta 18: 613-621

6. Benirschke K, Kaufmann P (2000) Pathology of the human placenta. Springer, New York, pp 947

7. Dunk C, Ahmed A (2000) Growth factor regulators of placental angiogenesis. In: Kingdom J, Baker PN (eds) Intrauterine growth restriction. Aetiology and management. Springer, London, pp 149-165

8. Wu P, Yonekura H, Li H et al. (2001) Hypoxia down-regulates endostatin production by human microvascular endothelial cells and pericytes. Biochem Biophys Res Commun 288: 1149-1154

9. Yamagishi S, Kobayashi K, Yamamoto H (1993) Vascular pericytes not only regulate growth, but also preserve prostacyclin-producing ability and protect against lipid peroxide-induced injury of co-cultured endothelial cells. Biochem Biophys Res Commun 190: 418-425

10. Darland DC, D'Amore PA (1999) Blood vessel maturation: vascular development comes of age. J Clin Invest 103: $157-158$

11. Speiser P, Gittelsohn AM, Patz A (1968) Studies on diabetic retinopathy. III. Influence of diabetes on intramural pericytes. Arch Ophthalmol 80: 332-337

12. Crocker DJ, Murad TM, Greer JC (1970) Role of the pericyte in wound healing. An ultrastructural study. Exp Mol Pathol 13: 51-65

13. Hellström M, Gerhardt H, Kalén M et al. (2001) Lack of pericytes leads to endothelial hyperplasia and abnormal vascular morphogenesis. J Cell Biol 153: 543-553

14. Sharkey AM, Cooper JC, Balmforth JR et al. (1996) Maternal plasma levels of vascular endothelial growth factor in normotensive pregnancies and in pregnancies complicated by pre-eclampsia. Eur J Clin Invest 26: 1182-1185 
15. Torry DS, Wang HS, Wang TH, Caudle MR, Torry RJ (1998) Preeclampsia is associated with reduced serum levels of placenta growth factor. Am J Obstet Gynecol 179: 1539-1544

16. Helske S, Vuorela P, Carpén O, Hornig C, Weich H, Halmesmäki E (2001) Expression of vascular endothelial growth factor receptors 1,2 and 3 in placentas from normal and complicated pregnancies. Mol Hum Reprod 7: 205210

17. Zhang EG, Smith SK, Baker PN, Charnock-Jones DS (2001) The regulation and localization of angiopoietin-1, -2 , and their receptor Tie2 in normal and pathologic human placentae. Mol Med 7: 624-635

18. Jackson MR, Mayhew TM, Haas JD (1987) Morphometric studies on villi in human term placentae and the effects of altitude, ethnic grouping and sex of newborn. Placenta 8: 487-495

19. Ali KZM, Burton GJ, Morad N, Ali ME (1996) Does hypercapillarization influence the branching pattern of terminal villi in the human placenta at high altitude? Placenta 17: 677-682

20. Burton GJ, Reshetnikova OS, Milovanov AP, Teleshova OV (1996) Stereological evaluation of vascular adaptations in human placental villi to differing forms of hypoxic stress. Placenta 17: 49-55

21. Macara LM, Kingdom JCP, Kaufmann P et al. (1996) Structural analysis of placental terminal villi from growthrestricted pregnancies with abnormal umbilical artery Doppler waveforms. Placenta 17: 37-48

22. Widness JA, Susa JB, Garcia JF et al. (1981) Increased erythropoiesis and elevated erythropoietin in infants born to diabetic mothers and in hyperinsulinemic Rhesus fetuses. J Clin Invest 67: 637-642

23. Mayhew TM, Sørensen FB, Klebe JG, Jackson MR (1993) Oxygen diffusive conductances in placentae from control and diabetic women. Diabetologia 36: 955-960

24. Mayhew TM, Sørensen FB, Klebe JG, Jackson MR (1994) Growth and maturation of villi in placentae from wellcontrolled diabetic women. Placenta 15: 57-65

25. Babawale MO, Lovat S, Mayhew TM, Lammiman MJ, James DK, Leach L (2000) Effects of gestational diabetes on junctional adhesion molecules in human term placental vasculature. Diabetologia 43: 1185-1196

26. Aiello LP, Avery RL, Arrigg PG et al. (1994) Vascular endothelial growth factor in ocular fluid of patients with diabetic retinopathy and other retinal disorders. New Engl J Med 331: 1480-1487

27. Malecaze F, Clemens S, Simorre-Pinatel V et al. (1994) Detection of vascular endothelial growth factor messenger RNA and vascular endothelial growth factor-like activity in proliferative diabetic retinopathy. Arch Ophthalmol 112: 1476-1482

28. Khaliq A, Foreman D, Ahmed A et al. (1998) Increased expression of placenta growth factor in proliferative diabetic retinopathy. Lab Invest 78: 109-116
29. Schweiki D, Itin A, Soffer D, Keshet E (1992) Vascular endothelial growth factor induced by hypoxia may mediate hypoxia-initiated angiogenesis. Nature 359: 843-845

30. Lu M, Kuroki M, Amano S et al. (1998) Advanced glycation end products increase retinal vascular endothelial growth factor expression. J Clin Invest 101: 1219-1224

31. Trein C, Giorgetti-Peraldi S, Murdaca J, Van Obberghen E (2001) Regulation of vascular endothelial growth factor expression by advanced glycation end products. J Biol Chem 276: 43836-43841

32. Okamoto T, Tanaka S, Stan AC et al. (2002) Advanced glycation end products induce angiogenesis in vivo. Microvasc Res 63: 186-195

33. White P (1949) Pregnancy complicating diabetes. Am J Med 7: 609-616

34. Diamond MP, Salyer SA, Vaughan WK et al. (1987) Re-assessment of White's classification and Pedersen's prognostically bad signs of diabetic pregnancies in insulindependent diabetic pregnancies. Am J Obstet Gynecol 156: 599-604

35. Howard CV, Reed MG (1998) Unbiased Stereology. Threedimensional measurement in microscopy. Bios Scientific, Oxford, pp 246

36. Teasdale F (1981) Histomorphometry of the placenta of the diabetic woman: class A diabetes mellitus. Placenta 2: $241-252$

37. Teasdale F (1983) Histomorphometry of the human placenta in class B diabetes mellitus. Placenta 4: 1-12

38. Teasdale F (1985) Histomorphometry of the human placenta in class $C$ diabetes mellitus. Placenta 6: 69-82

39. Björk O, Persson B (1982) Placental changes in relation to the degree of metabolic control in diabetes mellitus. Placenta 3: 367-378

40. Kadyrov M, Kosanke G, Kingdom J, Kaufmann P (1998) Increased fetoplacental angiogenesis during first trimester in anaemic women. Lancet 352: 1747-1749

41. Hovind P, Tarnow L, Oestergaard PB, Parving HH (2000) Elevated vascular endothelial growth factor in type 1 diabetic patients with diabetic nephropathy. Kidney Int Suppl 75: S56-S61

42. Beck L, D'Amore PA (1997) Vascular development: cellular and molecular regulation. FASEB J 11: 365-373

43. Hill DJ, Petrik J, Arany E (1998) Growth factors and the regulation of foetal growth. Diabetes Care 21 [Suppl 2]: B60-B69

44. Graier WF, Grubenthal I, Dittrich P, Wascher TC, Kostner GM (1995) Intracellular mechanism of high D-glucose-induced modulation of vascular cell proliferation. Eur J Pharmacol 294: 221-229

45. Kamal K, Du W, Mills I, Sumpio BE (1998) Antiproliferative effects of elevated glucose in human microvascular endothelial cells. J Cell Biochem 71: 491-501

46. Beltramo E, Pomero F, Allione A, D'Alù F, Ponte E, Porta M (2002) Pericyte adhesion is impaired on extracellular matrix produced by endothelial cells in high hexose concentrations. Diabetologia 45: 416-419 